Received: 15.03 .2021

Revised: 16.04 .2021

Accepted: 23.04 .2021

DOI: $10.17804 / 2410-9908.2021 .2 .052-063$

\title{
AN EXTREMUM PROBLEM FOR A LINEAR INTEGRO-DIFFERENTIAL SYSTEM DESCRIBING CREEPING FLOWS OF A VISCOELASTIC FLUID
}

\author{
M. A. Artemov ${ }^{\text {a) }}$ and E. S. Baranovskii ${ }^{\text {b)* }}$ \\ Voronezh State University, \\ 1 Universitetskaya Sq., Voronezh, 394018, Russian Federation \\ a) (iD http://orcid.org/0000-0001-8356-5418 $ه$ artemov_m_a@mail.ru; \\ b) iD http://orcid.org/0000-0002-1514-4475 esbaranovskii@gmail.com \\ *Corresponding author. E-mail: esbaranovskii@gmail.com \\ Address for correspondence: 1 Universitetskaya pl., Voronezh, 394018, Russian Federation
}

We consider an optimal control problem for an integro-differential system (with a quadratic cost functional) modeling a three-dimensional creeping flow of an incompressible viscoelastic fluid in a bounded domain with impermeable solid walls. The fluid flow is controlled by the timedependent external force. The concept of the control operator is proposed. We prove a theorem on the existence of a unique optimal control under the assumption that the set of admissible controls is convex and that it is closed in a suitable function space. Moreover, we obtain a variational inequality for the optimal control. The proof of this theorem is based on the application of the FaedoGalerkin approximation scheme taking into account energy estimates of approximate solutions and using the lemma on the existence and uniqueness of the metric projection of a point onto a closed convex set in a real Hilbert space.

Keywords: viscoelastic fluid, creeping flow, integro-differential equations, control operator, optimal control, existence theorem, variational inequality.

\section{References}

1. Fursikov A.V. Optimal Control of Distributed Systems. Providence, RI, AMS, 2000.

2. Litvinov W.G. Optimization in Elliptic Problems with Application to Mechanics of Deformable Body and Fluid Mechanics. Basel, Springer, 2000.

3. Alekseev G.V., Tereshko D.A. Analiz i optimizatsiya v gidrodinamike vyazkoi zhidkosti [Analysis and optimization in viscous fluid hydrodynamics]. Vladivostok, Dalnauka Publ., 2008. (In Russian).

4. Saut J.-C. Lectures on the mathematical theory of viscoelastic fluids. Lectures on the analysis of nonlinear partial differential equations, part 3, Int. Press, Somerville, 2013, pp. 325-393.

5. Temam R. Navier-Stokes Equations. Theory and Numerical Analysis, Amsterdam, New York, Oxford, North-Holland Publishing Co., 1977.

6. Oskolkov A.P., Shadiev R. Towards a theory of global solvability on $[0, \infty)$ of initialboundary value problems for the equations of motion of Oldroyd and Kelvin-Voight fluids. Journal of Mathematical Sciences, 1994, vol. 68, pp. 240-253. DOI: 10.1007/BF01249338.

7. Oskolkov A.P. Smooth global solutions of initial boundary-value problems for the equations of Oldroyd fluids and of their e-approximations. Journal of Mathematical Sciences, 1998, vol. 89, 1750-1763. DOI: 10.1007/BF02355375. 
8. Doubova A., Fernandez-Cara E. On the control of viscoelastic Jeffreys fluids. Systems \& Control Letters, 2012, vol. 61, pp. 573-579. DOI: 10.1016/j.sysconle.2012.02.003.

9. Hatzikiriakos S. G. Wall slip of molten polymers. Progress in Polymer Science, 2012, vol. 37, pp. 624-643. DOI: 10.1016/j.progpolymsci.2011.09.004.

10. Ullah H., Siddiqui A.M., Sun H., Haroon T. Slip effects on creeping flow of slightly nonNewtonian fluid in a uniformly porous slit. Journal of the Brazilian Society of Mechanical Sciences and Engineering, 2019, vol. 41, article ID 412. DOI: 10.1007/s40430-019-1917-2.

11. Burmasheva N.V., Prosviryakov E.Yu. An exact solution for describing the unidirectional Marangoni flow of a viscous incompressible fluid with the Navier boundary condition. Temperature field investigation. Diagnostics, Resource and Mechanics of materials and structures, 2020, iss. 1, pp. 6-23. DOI: 10.17804/2410-9908.2020.1.006-023.

12. Burmasheva N.V., Prosviryakov E.Yu. Exact solution for describing a unidirectional Marangoni flow of a viscous incompressible fluid with the Navier boundary condition. Pressure field investigation. Diagnostics, Resource and Mechanics of materials and structures, 2020, iss. 2, pp. 61-75. DOI: 10.17804/2410-9908.2020.2.061-075. Available at: https://dreamjournal.org/DREAM_Issue_2_2020_Burmasheva_N.V._et_al._061_075.pdf

13. Kinderlehrer D., Stampacchia G. An Introduction to Variational Inequalities and Their Applications. New York, Academic Press, 1980. 
Подана в журнал: 15.03.2021

УДК 517.9:532.1

DOI: $10.17804 / 2410-9908.2021 .2 .052-063$

\title{
ЭКСТРЕМАЛЬНАЯ ЗАДАЧА ДЛЯ ЛИНЕЙНОЙ ИНТЕГРО-ДИФФЕРЕНЦИАЛЬНОЙ СИСТЕМЫ, ОПИСЫВАЮЩЕЙ ПОЛЗУЩЕЕ ТЕЧЕНИЕ вЯЗКОУПРУГОЙ ЖИДКОСТИ
}

\author{
М. А. Артемов ${ }^{\text {a) }}$, Е. С. Барановский $)^{\text {(* }}$ \\ Воронежский государственный университет, \\ 1, Университетская пл, г. Воронеж, Российская Федераџия \\ a) (D) http://orcid.org/0000-0001-8356-5418 @artemov_m_a@mail.ru;

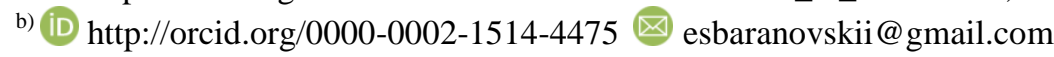 \\ *Ответственный автор. Электронная почта: esbaranovskii@gmail.com \\ Адрес для переписки: Университетская пл, 1, Воронеж, Российская Федерация
}

Рассматривается экстремальная задача управления для интегро-дифференциальной системы (с квадратичным целевым функционалом), моделирующей трехмерное ползущее течение несжимаемой вязкоупругой жидкости в ограниченной области с непроницаемыми твердыми стенками. Параметром управления служит зависящая от времени внешняя сила. Вводится понятие оператора управления. Доказана теорема о существовании единственного оптимального управления в предположении, что множество допустимых управлений выпукло и замкнуто в соответствующем функциональном пространстве. Кроме того, получено вариационное неравенство, которому удовлетворяет оптимальное управление. Доказательство теоремы основано на применении метода Фаэдо-Галеркина с учетом энергетических оценок приближенных решений и использовании леммы о существовании и единственности метрической проекции точки на выпуклое замкнутое множество в вещественном гильбертовом пространстве.

Ключевые слова: вязкоупругая жидкость, ползущее течение, интегро-дифференциальные уравнения, оператор управления, оптимальное управление, теорема существования, вариационные неравенства.

\section{1. Введение}

Проблемам оптимального управления в задачах гидродинамики посвящено много работ (см., например, [1-3] и приведенную там библиографию). При этом в большинстве из них рассматривается управление для классической системы Навье-Стокса, предназначенной для описания движения ньютоновской жидкости. Однако многие встречающиеся на практике среды не подчиняются ньютоновскому определяющему соотношению (закону трения Ньютона). К таким средам относятся, например, вязкоупругие жидкости с «памятью» [4], при математическом моделировании которых учитывается предыстория течения.

В настоящей статье изучается экстремальная задача управления для линейной интегро-дифференциальной системы, описывающей ползущее течение несжимаемой вязкоупругой среды с «памятью» в ограниченной трехмерной области $\Omega$ с границей $\partial \Omega$ на промежутке времени $[0, T]$ :

$$
\frac{\partial \mathbf{v}}{\partial t}-\mu \Delta \mathbf{v}-\int_{0}^{t} h(s, t) \Delta \mathbf{v}(\cdot, s) d s+\nabla \pi=\mathbf{f} \quad{ }_{8} Q_{T} ;
$$




$$
\begin{gathered}
\operatorname{div} \mathbf{v}=0{ }_{в} Q_{T} ; \\
\mathbf{v}=\mathbf{0} \quad \text { на } \partial \Omega \times(0, T) ; \\
\mathbf{v}(\cdot, 0)=\mathbf{0} \quad{ }_{в} \Omega ; \\
\mathbf{f} \in \mathbf{F} ; \\
J_{\xi}(\mathbf{v}, \mathbf{f})=\xi\|\mathbf{v}-\mathbf{b}\|_{\mathbf{L}^{2}\left(Q_{T}\right)}^{2}+(1-\xi)\|\mathbf{f}\|_{\mathbf{L}^{2}\left(Q_{T}\right)}^{2} \rightarrow \min ,
\end{gathered}
$$

где $Q_{T}:=\Omega \times(0, T) ; \mathbf{v}: \bar{Q}_{T} \rightarrow \mathbf{R}^{3}-$ вектор-функция скорости; $\pi: \bar{Q}_{T} \rightarrow \mathbf{R}-$ функция давления; $\mu>0-$ коэффициент вязкости; $h:[0, T] \times[0, T] \rightarrow[0, \infty)-$ ядро вязкоупругости; $\mathbf{f}: \bar{Q}_{T} \rightarrow \mathbf{R}^{3}-$ внешняя сила (управляющая функция); $\mathbf{F}-$ множество допустимых управлений; $J_{\mathrm{o}}$ - «компромиссный» целевой функционал [1] с весовым коэффициентом $\xi \in(0,1)$; $\mathbf{b}: \bar{\Omega} \rightarrow \mathbf{R}^{3}$ - заданная вектор-функция (желаемое поле скоростей); операторы $\nabla, \Delta$ и $\operatorname{div}$ обозначают соответственно градиент, лапласиан и дивергенцию по пространственным переменным $x_{1}, x_{2}, x_{3}$.

В вырожденном случае $h(s, t) \equiv 0$ приходим к линеаризованным уравнениям НавьеСтокса [5]. Если $h(s, t) \equiv \beta \exp (\alpha(s-t))$, то система (1), (2) сводится к линеаризованным уравнениям движения вязкоупругой жидкости типа Джеффриса-Олдройда $[6,7]$. Аппроксимативная управляемость соответствующих уравнений установлена в [8].

В настоящей статье мы не будем ограничиваться конкретным выбором ядра вязкоупругости - рассмотрим общую ситуацию, предполагая лишь, что функция $h$ непрерывна. Основная цель работы заключается в том, чтобы установить однозначную разрешимость экстремальной задачи (1)-(6) в классе слабых решений. Доказательство основано на построении приближенных решений по методу Фаэдо-Галеркина, выводе энергетических оценок, изучении свойств оператора управления и применении леммы о существовании и единственности метрической проекции точки на выпуклое замкнутое множество в вещественном гильбертовом пространстве.

Отметим, что результаты работы могут быть перенесены на случай граничных условий проскальзывания, учет которых особенно важен при моделировании потоков неньютоновских сред $[9,10]$ и в задачах термоконвекции $[11,12]$.

\section{2. Вариационная формулировка задачи и основной результат работы}

Условимся использовать стандартные обозначения для пространства Лебега $\mathbf{L}^{2}(\Omega)$ и пространства Соболева $\mathbf{W}^{1,2}(\Omega)$ векторных функций, заданных на области $\Omega$ и со значениями в $\mathbf{R}^{3}$ (или в $\mathbf{R}^{3 \times 3}$, что понятно из контекста). Скалярное произведение в $\mathbf{L}^{2}(\Omega)$ будем обозначать с помощью круглых скобок, т. е. $(\mathbf{u}, \mathbf{w})=(\mathbf{u}, \mathbf{w})_{\mathbf{L}}^{2}(\Omega)$.

Следуя [5, гл. I], введем пространства:

$\mathbf{Y}:=\left\{\mathbf{w} \in \mathbf{C}^{\infty}(\Omega): \operatorname{supp} \mathbf{w} \subset \Omega, \operatorname{div} \mathbf{w} \equiv 0\right\}$,

$\mathbf{H}$ - замыкание $\mathbf{Y}$ в пространстве $\mathbf{L}^{2}(\Omega)$,

$\mathbf{V}$ - замыкание $\mathbf{Y}$ в пространстве $\mathbf{W}^{1,2}(\Omega)$.

Снабдим пространство $\mathbf{V}$ скалярным произведением 


$$
(\mathbf{u}, \mathbf{w})_{\mathbf{V}}:=(\nabla \mathbf{u}, \nabla \mathbf{w})
$$

и соответствующей евклидовой нормой

$$
\|\mathbf{u}\|_{\mathbf{v}}:=(\nabla \mathbf{u}, \nabla \mathbf{u})^{1 / 2}
$$

Заметим, что норма $\|\cdot\|_{\mathbf{V}}$ эквивалентна стандартной норме $\|\cdot\|_{\mathbf{W}^{1,2}(\Omega)}$.

Функции, зависящие от переменных $\mathbf{x} \in \Omega$ и $t \in(0, T)$, будем рассматривать так же, как функции аргумента $t$ со значениями в банаховом пространстве, определенном на $\Omega$. Например, $\mathbf{L}^{2}\left(0, T ; \mathbf{L}^{2}(\Omega)\right)$ - это пространство функций, заданных на $(0, T)$ и действующих в $\mathbf{L}^{2}(\Omega)$. Очевидно, что

$$
\mathbf{L}^{2}\left(0, T ; \mathbf{L}^{2}(\Omega)\right)=\mathbf{L}^{2}\left(Q_{T}\right)
$$

Предположим, что выполнены следующие условия:

(i) граница области $\Omega$ является локально-липшицевой;

(ii) функция $h:[0, T] \times[0, T] \rightarrow[0, \infty)$ непрерывна;

(iii) выполнено включение $\mathbf{b} \in \mathbf{L}^{2}(0, T ; \mathbf{H})$;

(iv) множество $\mathbf{F}$ выпукло и замкнуто в $\mathbf{L}^{2}\left(0, T ; \mathbf{L}^{2}(\Omega)\right)$.

Определение 1. Допустимой парой для системы (1)-(6) будем называть пару векторфункций (f, v):

$$
\mathbf{f} \in \mathbf{F}, \quad \mathbf{v} \in \mathbf{L}^{2}(0, T ; \mathbf{V}) \cap \mathbf{C}([0, T] ; \mathbf{H}), \quad \mathbf{v}(\cdot, 0)=\mathbf{0},
$$

и для любой вектор-функции $\mathbf{w} \in \mathbf{V}$ выполнено следующее равенство:

$$
\frac{d}{d t}(\mathbf{v}, \mathbf{w})+\mu(\nabla \mathbf{v}, \nabla \mathbf{w})+\left(\int_{0}^{t} h(s, t) \nabla \mathbf{v}(\cdot, s) d s, \nabla \mathbf{w}\right)=(\mathbf{f}, \mathbf{w}),
$$

где оператор $d / d t$ обозначает обобщенную производную по $t$.

Множество всех допустимых пар обозначим через $\mathbf{M ( F )}$.

Для любого управления $\mathbf{f} \in \mathbf{F}$ существует единственная пара $(\mathbf{f}, \mathbf{v})$, принадлежащая множеству $\mathbf{M ( F )}$ (разд. 3). Это позволяет корректно определить оператор управления $\mathbf{K}$.

Oпр

$$
\mathbf{K}: \mathbf{F} \subset \mathbf{L}^{2}\left(0, T ; \mathbf{L}^{2}(\Omega)\right) \rightarrow \mathbf{L}^{2}(0, T ; \mathbf{H}), \quad \mathbf{K}(\mathbf{f})=\mathbf{v},
$$

где $\mathbf{f} \in \mathbf{F}$ и $\mathbf{v}-$ вторая компонента пары $(\mathbf{f}, \mathbf{v}) \in \mathbf{M}(\mathbf{F})$.

Через $\mathbf{G}(\mathbf{K})$ обозначим график оператора $\mathbf{K}$, т. е.

$$
\mathbf{G}(\mathbf{K}):=\{(\mathbf{f}, \mathbf{K}(\mathbf{f})): \mathbf{f} \in \mathbf{F}\} \subset \mathbf{L}^{2}\left(0, T ; \mathbf{L}^{2}(\Omega)\right) \times \mathbf{L}^{2}(0, T ; \mathbf{H}) .
$$

Определение 3. Вектор-функция $\mathbf{f}_{*} \in \mathbf{F}$ является оптимальным управлением в системе (1)-(6), если 


$$
\begin{gathered}
\xi\left\|\mathbf{K}\left(\mathbf{f}_{*}\right)-\mathbf{b}\right\|_{\mathbf{L}^{2}(0, T ; \mathbf{H})}^{2}+(1-\xi)\left\|\mathbf{f}_{*}\right\|_{\mathbf{L}^{2}\left(0, T ; \mathbf{L}^{2}(\Omega)\right)}^{2}= \\
=\inf \left\{\xi\|\mathbf{K}(\mathbf{f})-\mathbf{b}\|_{\mathbf{L}^{2}(0, T ; \mathbf{H})}^{2}+(1-\xi) \|\left.\mathbf{f}\right|_{\mathbf{L}^{2}\left(0, T ; \mathbf{L}^{2}(\Omega)\right)} ^{2}: \mathbf{f} \in \mathbf{F}\right\} .
\end{gathered}
$$

Основной результат работы сформулирован в следующей теореме.

Tеорема. Пусть выполнень условия (i)-(iv). Тогда в системе (1)-(6) существует единственное оптимальное управление. При этом вектор-функция $\mathbf{f}_{*} \in \mathbf{F}$ является оптимальным управлением тогда и только тогда, когда выполнено вариационное неравенство

$$
\xi\left(\mathbf{K}\left(\mathbf{f}_{*}\right)-\mathbf{b}, \mathbf{K}(\mathbf{f})-\mathbf{K}\left(\mathbf{f}_{*}\right)\right)_{\mathbf{L}^{2}(0, T ; \mathbf{H})} \geq(1-\xi)\left(\mathbf{f}_{*}, \mathbf{f}_{*}-\mathbf{f}\right){ }_{\mathbf{L}^{2}\left(0, T ; \mathbf{L}^{2}(\Omega)\right)} \forall \mathbf{f} \in \mathbf{F} .
$$

Доказательство этого утверждения представлено в разд. 4.

\section{3. Обоснование корректности определения оператора управления $\mathrm{K}$}

Установим некоторые свойства допустимых пар.

Лемма 1. Если $\left(\mathbf{f}_{1}, \mathbf{v}_{1}\right) \in \mathbf{M}(\mathbf{F}) u\left(\mathbf{f}_{2}, \mathbf{v}_{2}\right) \in \mathbf{M}(\mathbf{F})$, тогда

$$
\begin{gathered}
\max _{\tau \in[0, T]}\left(\left\|\mathbf{v}_{1}(\cdot, \tau)-\mathbf{v}_{2}(\cdot, \tau)\right\|^{2}+\mu \int_{0}^{\tau} \| \mathbf{v}_{1}(\cdot, s)-\left.\mathbf{v}_{2}(\cdot, s)\right|_{\mathbf{V}} ^{2} d s\right) \leq \\
\leq \frac{e^{C(h, T, \mu) T}}{C(h, T, \mu)}\left\|\mathbf{f}_{1}-\mathbf{f}_{2}\right\|_{\mathbf{L}^{2}\left(0, T ; \mathbf{L}^{2}(\Omega)\right)}^{2},
\end{gathered}
$$

где

$$
C(h, T, \mu):=\frac{1}{\mu^{2}} \max _{t \in[0, T]} \int_{0}^{t} h^{2}(t, s) d s .
$$

Доказательство. Отождествляя гильбертово пространство Н и сопряженное к нему $\mathbf{H}^{*}$ в соответствии с теоремой представления Рисса, приходим к цепочке вложений

$$
\mathbf{V} \subset \mathbf{H} \cong \mathbf{H}^{*} \subset \mathbf{V}^{*}
$$

Заметим, что имеют место включения

$$
\frac{d \mathbf{v}_{i}}{d t} \in \mathbf{L}^{2}\left(0, T ; \mathbf{V}^{*}\right), \quad i=1,2
$$

и применим лемму 1.2 [5, гл. III, §1] к вектор-функции $\mathbf{v}_{1}-\mathbf{v}_{2}$. Получаем, что для п. в. $t \in(0, T)$ выполнено равенство 


$$
\frac{d}{d t}\left\|\mathbf{v}_{1}-\mathbf{v}_{2}\right\|^{2}=2\left\langle\frac{d\left(\mathbf{v}_{1}-\mathbf{v}_{2}\right)}{d t}, \mathbf{v}_{1}-\mathbf{v}_{2}\right\rangle_{\mathbf{v}^{*} \times \mathbf{v}},
$$

где угловые скобки $\langle\cdot,\rangle \mathbf{V}^{*} \times \mathbf{v}$ обозначают отношение двойственности между пространством $\mathbf{V}$ и его сопряженным $\mathbf{V}^{*}$.

Поскольку

$$
\left(\mathbf{f}_{i}, \mathbf{v}_{i}\right) \in \mathbf{M}(\mathbf{F}), \quad i=1,2
$$

легко видеть, что

$$
\begin{gathered}
\left\langle\frac{d\left(\mathbf{v}_{1}-\mathbf{v}_{2}\right)}{d t}, \mathbf{w}\right\rangle_{\mathbf{v}^{*} \times \mathbf{V}}+\mu\left(\nabla\left(\mathbf{v}_{1}-\mathbf{v}_{2}\right), \nabla \mathbf{w}\right)_{+} \\
+\left(\int_{0}^{t} h(s, t) \nabla\left(\mathbf{v}_{1}(\cdot, s)-\mathbf{v}_{2}(\cdot, s)\right) d s, \nabla \mathbf{w}\right)=\left(\mathbf{f}_{1}-\mathbf{f}_{2}, \mathbf{w}\right),
\end{gathered}
$$

для любой вектор-функции $\mathbf{w} \in \mathbf{V}$.

Подставляя $\mathbf{w}:=\mathbf{v}_{1}(\cdot, t)-\mathbf{v}_{2}(\cdot, t)$ в равенство (10) с учетом (9), приходим к следующему равенству:

$$
\begin{gathered}
\frac{d}{d t}\left\|\mathbf{v}_{1}(\cdot, t)-\mathbf{v}_{2}(\cdot, t)\right\|^{2}+2 \mu\left\|\mathbf{v}_{1}(\cdot, t)-\mathbf{v}_{2}(\cdot, t)\right\|_{\mathbf{v}^{+}}^{2} \\
+2\left(\int_{0}^{t} h(s, t) \nabla\left(\mathbf{v}_{1}(\cdot, s)-\mathbf{v}_{2}(\cdot, s)\right) d s, \nabla\left(\mathbf{v}_{1}(\cdot, t)-\mathbf{v}_{2}(\cdot, t)\right)\right)= \\
=2\left(\mathbf{f}_{1}(\cdot, t)-\mathbf{f}_{2}(\cdot, t), \mathbf{v}_{1}(\cdot, t)-\mathbf{v}_{2}(\cdot, t)\right),
\end{gathered}
$$

Затем, применяя неравенства Гельдера и Юнга, получаем:

$$
\begin{gathered}
\frac{d}{d t}\left\|\mathbf{v}_{1}(\cdot, t)-\mathbf{v}_{2}(\cdot, t)\right\|^{2}+2 \mu\left\|\mathbf{v}_{1}(\cdot, t)-\mathbf{v}_{2}(\cdot, t)\right\|_{\mathbf{V}}^{2} \leq \\
\leq \mu\left\|\mathbf{v}_{1}(\cdot, t)-\mathbf{v}_{2}(\cdot, t)\right\|_{\mathbf{v}}^{2}+C(h, T, \mu) \mu \int_{0}^{t}\left\|\mathbf{v}_{1}(\cdot, s)-\mathbf{v}_{2}(\cdot, s)\right\|_{\mathbf{v}}^{2} d s+ \\
+\frac{\left\|\mathbf{f}_{1}(\cdot, t)-\mathbf{f}_{2}(\cdot, t)\right\|^{2}}{C(h, T, \mu)}+C(h, T, \mu)\left\|\mathbf{v}_{1}(\cdot, t)-\mathbf{v}_{2}(\cdot, t)\right\|^{2}
\end{gathered}
$$

После элементарных преобразований получаем

$$
\begin{gathered}
\frac{d}{d t}\left\|\mathbf{v}_{1}(\cdot, t)-\mathbf{v}_{2}(\cdot, t)\right\|^{2}+\mu\left\|\mathbf{v}_{1}(\cdot, t)-\mathbf{v}_{2}(\cdot, t)\right\|_{\mathbf{v}}^{2} \leq \\
\leq C(h, T, \mu)\left(\left\|\mathbf{v}_{1}(\cdot, t)-\mathbf{v}_{2}(\cdot, t)\right\|^{2}+\mu \int_{0}^{t} \| \mathbf{v}_{1}(\cdot, s)-\left.\mathbf{v}_{2}(\cdot, s)\right|_{\mathbf{v}} ^{2} d s\right)+\frac{\left\|\mathbf{f}_{1}(\cdot, t)-\mathbf{f}_{2}(\cdot, t)\right\|^{2}}{C(h, T, \mu)} .
\end{gathered}
$$


Проинтегрируем обе части последнего неравенства по $t$ от 0 до $\tau$ :

$$
\begin{gathered}
\left\|\mathbf{v}_{1}(\cdot, \tau)-\mathbf{v}_{2}(\cdot, \tau)\right\|^{2}+\mu \int_{0}^{\tau}\left\|\mathbf{v}_{1}(\cdot, s)-\mathbf{v}_{2}(\cdot, s)\right\|_{\mathbf{v}}^{2} d s \leq \\
\leq C(h, T, \mu) \int_{0}^{\tau}\left(\left|\mathbf{v}_{1}(\cdot, t)-\mathbf{v}_{2}(\cdot, t)\right|^{2}+\mu \int_{0}^{t} \| \mathbf{v}_{1}(\cdot, s)-\mathbf{v}_{2}(\cdot, s) \mid \mathbf{v}_{\mathbf{v}}^{2} d s\right) d t+ \\
+\frac{1}{C(h, T, \mu)} \int_{0}^{\tau}\left\|\mathbf{f}_{1}(\cdot, t)-\mathbf{f}_{2}(\cdot, t)\right\|^{2} d t
\end{gathered}
$$

Применяя неравенство Гронуолла, получаем следующую оценку:

$$
\left\|\mathbf{v}_{1}(\cdot, \tau)-\mathbf{v}_{2}(\cdot, \tau)\right\|^{2}+\mu \int_{0}^{\tau}\left\|\mathbf{v}_{1}(\cdot, s)-\left.\mathbf{v}_{2}(\cdot, s)\right|_{\mathbf{v}} ^{2} d s \leq \frac{e^{C(h, T, \mu) T}}{C(h, T, \mu)}\right\| \mathbf{f}_{1}-\mathbf{f}_{2} \|_{\mathbf{L}^{2}\left(0, T ; \mathbf{L}^{2}(\Omega)\right)}^{2},
$$

откуда и вытекает требуемое неравенство (8).

Лемма 2. Для любой вектор-функции $\mathbf{f}_{0} \in \mathbf{F}$ существует единственная векторфункция $\mathbf{v}_{0}$ такая, что $\left(\mathbf{f}_{0}, \mathbf{v}_{0}\right) \in \mathbf{M}(\mathbf{F})$.

Доказательство. Для нахождения $\mathbf{v}_{0}$ построим последовательность приближенных решений $\left\{\mathbf{v}_{n}\right\}_{n=1}^{\infty}$ по методу Фаэдо-Галеркина и затем осуществим предельный переход при $n \rightarrow \infty$, используя равномерную ограниченность норм $\left\{\left\|\mathbf{v}_{n}\right\|_{\mathbf{L}}^{2}(0, T ; \mathbf{V})\right\}_{n=1}^{\infty}$.

Приближенные решения, соответствующие выбору $\mathbf{f}=\mathbf{f}_{0}$, представим в виде сумм:

$$
\mathbf{v}_{n}(\mathbf{x}, t):=\sum_{j=1}^{n} a_{n j}(t) \mathbf{w}_{j}(\mathbf{x}), \quad \mathbf{x} \in \Omega, t \in(0, T),
$$

где $a_{n j}:[0, T] \rightarrow \mathbf{R}-$ неизвестные функции; $\left\{\mathbf{w}_{j}\right\}_{j=1}^{\infty}-$ полная последовательность в $\mathbf{V} ;$ образующая ортонормированный базис в $\mathbf{H}$.

Зафиксируем произвольное натуральное число $n$. Рассмотрим задачу Коши:

$$
\begin{gathered}
\left(\frac{\partial \mathbf{v}_{n}}{\partial t}, \mathbf{w}_{k}\right)+\mu\left(\nabla \mathbf{v}_{n}, \nabla \mathbf{w}_{k}\right)+\left(\int_{0}^{t} h(t, s) \nabla \mathbf{v}_{n}(s) d s, \nabla \mathbf{w}_{k}\right)= \\
=\left(\mathbf{f}_{0}, \mathbf{w}_{k}\right), \quad t \in(0, T), k \in\{1, \ldots, n\}, \\
\mathbf{v}_{n}(\mathbf{x}, 0)=\mathbf{0}, \quad \mathbf{x} \in \Omega .
\end{gathered}
$$

Линейная интегро-дифференциальная система (11) с начальными данными (12) единственным образом определяет функции $a_{n k}$ на всем отрезке $[0, T]$.

Выведем независящие от параметра $n$ оценки решений задачи (11), (12). Предположим, что $\mathbf{v}_{n}$ удовлетворяет равенствам (11) и (12). Умножим обе части (11) на $a_{n k}(t)$. Сложив полученные равенства по $k=1, \ldots, n$, находим: 


$$
\left(\frac{\partial \mathbf{v}_{n}}{\partial t}, \mathbf{v}_{n}\right)+\mu\left(\nabla \mathbf{v}_{n}, \nabla \mathbf{v}_{n}\right)+\left(\int_{0}^{t} h(t, s) \nabla \mathbf{v}_{n}(s) d s, \nabla \mathbf{v}_{n}\right)=\left(\mathbf{f}_{0}, \mathbf{v}_{n}\right), \quad t \in(0, T) .
$$

Умножим обе части последнего равенства на 2. После элементарных преобразований находим, что

$$
\frac{d}{d t}\left\|\mathbf{v}_{n}\right\|^{2}+2 \mu\left\|\mathbf{v}_{n}\right\|_{\mathbf{v}}^{2}+2\left(\int_{0}^{t} h(t, s) \nabla \mathbf{v}_{n}(s) d s, \nabla \mathbf{v}_{n}\right)=2\left(\mathbf{f}_{0}, \mathbf{v}_{n}\right), \quad t \in(0, T) .
$$

Применяя неравенства Гельдера и Юнга, получаем

$$
\begin{gathered}
\frac{d}{d t}\left\|\mathbf{v}_{n}\right\|^{2}+2 \mu\left\|\mathbf{v}_{n}\right\|_{\mathbf{v}}^{2} \leq \mu\left\|\mathbf{v}_{n}\right\|_{\mathbf{v}}^{2}+C(h, T, \mu) \mu \int_{0}^{t}\left\|\mathbf{v}_{n}(s)\right\|_{\mathbf{v}}^{2} d s+ \\
+\frac{1}{C(h, T, \mu)}\left\|\mathbf{f}_{0}\right\|^{2}+C(h, T, \mu)\left\|\mathbf{v}_{n}\right\|^{2}
\end{gathered}
$$

откуда следует:

$$
\frac{d}{d t}\left\|\mathbf{v}_{n}\right\|^{2}+\mu\left\|\mathbf{v}_{n}\right\|_{\mathbf{v}}^{2} \leq C(h, T, \mu)\left(\left\|\mathbf{v}_{n}\right\|^{2}+\mu \int_{0}^{t}\left\|\mathbf{v}_{n}(s)\right\|_{\mathbf{V}}^{2} d s\right)+\frac{1}{C(h, T, \mu)}\left\|\mathbf{f}_{0}\right\|^{2} .
$$

Проинтегрируем обе части (13) по $t$ от 0 до $\tau$ :

$$
\begin{gathered}
\left\|\mathbf{v}_{n}(\cdot, \tau)\right\|^{2}+\mu \int_{0}^{\tau}\left\|\mathbf{v}_{n}(\cdot, s)\right\|_{\mathbf{V}}^{2} d s \leq \\
\leq C_{2}(h, T, \mu) \int_{0}^{\tau}\left(\left\|\mathbf{v}_{n}(\cdot, t)\right\|^{2}+\mu \int_{0}^{t}\left\|\mathbf{v}_{n}(s)\right\|_{\mathbf{V}}^{2} d s\right) d t+\frac{1}{C(h, T, \mu)} \int_{0}^{\tau}\left\|\mathbf{f}_{0}(\cdot, t)\right\|^{2} d t,
\end{gathered}
$$

откуда с помощью неравенства Гронуолла выводим оценку

$$
\max _{\tau \in[0, T]}\left(\left\|\mathbf{v}_{n}(\cdot, \tau)\right\|^{2}+\mu \int_{0}^{\tau}\left\|\mathbf{v}_{n}(\cdot, s)\right\|_{\mathbf{V}}^{2} d s\right) \leq \frac{e^{C(h, T, \mu) T}}{C(h, T, \mu)}\left\|\mathbf{f}_{0}\right\|_{\mathbf{L}^{2}\left(0, T ; \mathbf{L}^{2}(\Omega)\right)}^{2} .
$$

Из неравенства (14) следует равномерная по $n$ ограниченность норм $\left\{\left\|\mathbf{v}_{n}\right\|_{\mathbf{L}^{2}(0, T ; \mathbf{V})}\right\}_{n=1}^{\infty}$. Поэтому, переходя к подпоследовательности (если это необходимо), получаем:

$$
\mathbf{v}_{n} \rightarrow \mathbf{v}_{0} \text { слабо в } \mathbf{L}^{2}(0, T ; \mathbf{V})
$$

для некоторой вектор-функции $\mathbf{v}_{0} \in \mathbf{L}^{2}(0, T ; \mathbf{V})$.

Умножим обе части равенства (11) на произвольную $C^{\infty}$-гладкую функцию $\eta$ с носителем, содержащимся в интервале $(0, T)$, и проинтегрируем по $t$ от 0 до $T$. Применив интегрирование по частям к первому слагаемому из левой части полученного равенства, приходим к соотношению: 


$$
\begin{gathered}
-\int_{0}^{T}\left(\mathbf{v}_{n}, \mathbf{w}_{k}\right) \eta^{\prime} d t+\mu \int_{0}^{T}\left(\nabla \mathbf{v}_{n}, \nabla \mathbf{w}_{k}\right) \eta d t+ \\
+\int_{0}^{T}\left(\int_{0}^{t} h(t, s) \nabla \mathbf{v}_{n}(s) d s, \nabla \mathbf{w}_{k}\right) \eta d t=\int_{0}^{T}\left(\mathbf{f}_{0}, \mathbf{w}_{k}\right) \eta d t, \quad k \in\{1, \ldots, n\} .
\end{gathered}
$$

Используя слабую сходимость (15), перейдем к пределу при $n \rightarrow \infty$ в (16):

$$
\begin{gathered}
-\int_{0}^{T}\left(\mathbf{v}_{0}, \mathbf{w}_{k}\right) \eta^{\prime} d t+\mu \int_{0}^{T}\left(\nabla \mathbf{v}_{0}, \nabla \mathbf{w}_{k}\right) \eta d t+ \\
+\int_{0}^{T}\left(\int_{0}^{t} h(t, s) \nabla \mathbf{v}_{0}(s) d s, \nabla \mathbf{w}_{k}\right) \eta d t=\int_{0}^{T}\left(\mathbf{f}_{0}, \mathbf{w}_{k}\right) \eta d t, \quad k \in\{1,2, \ldots\} .
\end{gathered}
$$

Поскольку последовательность $\left\{\mathbf{w}_{j}\right\}_{j=1}^{\infty}$ полна в $\mathbf{V}$, последнее равенство останется справедливым, если заменить $\mathbf{w}_{k}$ на произвольную вектор-функцию $\mathbf{w} \in \mathbf{V}$. Отсюда, в частности, следует:

$$
\frac{d \mathbf{v}_{0}}{d t} \in \mathbf{L}^{2}\left(0, T ; \mathbf{V}^{*}\right)
$$

Поэтому, вновь применяя лемму 1.2 [5, гл. III, $\S 1]$, получаем:

$$
\mathbf{v}_{0} \in \mathbf{C}([0, T] ; \mathbf{H})
$$

Принимая во внимание (12), нетрудно проверить, что выполнено начальное условие $\mathbf{v}_{0}(\cdot, 0)=\mathbf{0}$. Таким образом, установлено, что $\left(\mathbf{f}_{0}, \mathbf{v}_{0}\right) \in \mathbf{M}(\mathbf{F})$.

Единственность вектор-функции $\mathbf{v}_{0}$, удовлетворяющей условиям леммы 2, непосредственно следует из леммы 1.

Следствие. Оператор управления $\mathbf{K}$ определен корректно и непрерывен как отображение из подмножества $\mathbf{F}$ пространства $\mathbf{L}^{2}\left(0, T ; \mathbf{L}^{2}(\Omega)\right)$ в пространство $\mathbf{L}^{2}(0, T ; \mathbf{H})$.

\section{4. Доказательство основного результата}

Нам понадобятся следующие две леммы [13, гл. I, §2].

Лемма 3. Пусть $\mathbf{S}$ - выпуклое замкнутое подмножество гильбертова пространства $\mathbf{U}$. Тогда для каждого $\mathbf{q} \in \mathbf{U}$ существует единственная точка $\mathbf{p} \in \mathbf{U}$ :

$$
\|\mathbf{q}-\mathbf{p}\|_{\mathbf{U}}=\inf \left\{\|\mathbf{q}-\mathbf{s}\|_{\mathbf{u}}: \mathbf{s} \in \mathbf{S}\right\}
$$

Замечание. Точка р, удовлетворяющая соотношению (17), называется метрической проекиией точки $\mathbf{q}$ на множество $\mathbf{S}$. При этом используется обозначение $\mathbf{p}=\operatorname{Pr}_{\mathbf{S}} \mathbf{q}$.

Лемма 4. Пусть $\mathbf{S}$ - выпуклое замкнутое подмножество гильбертова пространства $\mathbf{U} u \mathbf{q} \in \mathbf{U}$. Тогда $\mathbf{p}=\operatorname{Pr}_{\mathbf{S}} \mathbf{q}$ в том и только в том случае, когда 


$$
(\mathbf{p}, \mathbf{S}-\mathbf{p})_{\mathbf{U}} \geq(\mathbf{q}, \mathbf{s}-\mathbf{p})_{\mathbf{U}}
$$

для любого $\mathbf{S} \in \mathbf{S}$.

Доказательство теоремы. Заметим, что $\mathbf{f}_{*} \in \mathbf{F}$ - оптимальное управление в системе (1)-(6) тогда и только тогда, когда пара функций $\left(\mathbf{f}_{*}, \mathbf{K}\left(\mathbf{f}_{*}\right)\right)$ является метрической проекцией пары $(\mathbf{0}, \mathbf{b})$ на график оператора управления $\mathbf{K}$ в декартовом произведении $\mathbf{L}^{2}\left(0, T ; \mathbf{L}^{2}(\Omega)\right) \times \mathbf{L}^{2}(0, T ; \mathbf{H})$ со скалярным произведением:

$$
(\mathbf{f}, \mathbf{g})_{\mathbf{L}^{2}\left(0, T ; \mathbf{L}^{2}(\Omega)\right) \times \mathbf{L}^{2}(0, T ; \mathbf{H})}:=(1-\xi)\left(\mathbf{f}_{1}, \mathbf{g}_{1}\right)_{\mathbf{L}^{2}\left(0, T ; \mathbf{L}^{2}(\Omega)\right)}+\xi\left(\mathbf{f}_{2}, \mathbf{g}_{2}\right){ }_{\mathbf{L}}{ }^{2}(0, T ; \mathbf{H}),
$$

где $\mathbf{f}=\left(\mathbf{f}_{1}, \mathbf{f}_{2}\right)$ и $\mathbf{g}=\left(\mathbf{g}_{1}, \mathbf{g}_{2}\right)$.

Пусть

$$
\mathbf{U}:=\mathbf{L}^{2}\left(0, T ; \mathbf{L}^{2}(\Omega)\right) \times \mathbf{L}^{2}(0, T ; \mathbf{H}), \quad \mathbf{S}:=\mathbf{G}(\mathbf{K}), \quad \mathbf{q}:=(\mathbf{0}, \mathbf{b}) .
$$

Применяя лемму 3, получаем метрическую проекцию $\mathbf{p}=\left(\mathbf{f}_{*}, \mathbf{K}\left(\mathbf{f}_{*}\right)\right)$ точки $\mathbf{q}$ на множество S. При том, что $\mathbf{f}_{*}$ - единственное оптимальное управление в системе (1)-(6).

Принимая во внимание (19) и равенство $\mathbf{p}=\left(\mathbf{f}_{*}, \mathbf{K}\left(\mathbf{f}_{*}\right)\right)$, выводим из (18) вариационное неравенство (7). Теорема доказана.

\section{5. Заключение}

В статье установлена однозначная разрешимость экстремальной задачи управления для линейной интегро-дифференциальной системы, описывающей трехмерное ползущее течение несжимаемой вязкоупругой жидкости в ограниченной области с непроницаемыми твердыми стенками. Выведено вариационное неравенство, характеризующее оптимальное управление. В дальнейшем предполагается развить предложенный подход и получить новые результаты для нелинейных моделей вязкоупругих сред с «памятью» при учете инерционных сил и тепловых эффектов.

\section{Литература}

1. Fursikov A. V. Optimal Control of Distributed Systems, Rus. transl. - Providence, RI, AMS, 2000 .

2. Litvinov W. G. Optimization in Elliptic Problems with Application to Mechanics of Deformable Body and Fluid Mechanics. - Basel, Springer, 2000.

3. Алексеев Г. В., Терешко Д. А. Анализ и оптимизация в гидродинамике вязкой жидкости. - Владивосток : Дальнаука, 2008.

4. Saut J.-C. Lectures on the mathematical theory of viscoelastic fluids. Lectures on the analysis of nonlinear partial differential equations. Part 3. - Int. Press, Somerville, 2013. - P. 325-393.

5. Temam R. Navier-Stokes Equations. Theory and Numerical Analysis. - Amsterdam, New York, Oxford : North-Holland Publishing Co., 1977.

6. Oskolkov A. P., Shadiev R. Towards a theory of global solvability on $[0, \infty)$ of initialboundary value problems for the equations of motion of Oldroyd and Kelvin-Voight fluids // Journal of Mathematical Sciences. - 1994. - Vol. 68. - P. 240-253. - DOI: 10.1007/BF01249338.

7. Oskolkov A. P. Smooth global solutions of initial boundary-value problems for the equations of Oldroyd fluids and of their e-approximations // Journal of Mathematical Sciences. - 1998. Vol. 89. - P. 1750-1763. - DOI: 10.1007/BF02355375. 
8. Doubova A., Fernandez-Cara E. On the control of viscoelastic Jeffreys fluids // Systems \& Control Letters. - 2012. - Vol. 61. - P. 573-579. - DOI: 10.1016/j.sysconle.2012.02.003.

9. Hatzikiriakos S. G. Wall slip of molten polymers // Progress in Polymer Science. - 2012. Vol. 37. - P. 624-643. - DOI: 10.1016/j.progpolymsci.2011.09.004.

10. Slip effects on creeping flow of slightly non-Newtonian fluid in a uniformly porous slit / H. Ullah, A. M. Siddiqui, H. Sun, T. Haroon // Journal of the Brazilian Society of Mechanical Sciences and Engineering. - 2019. - Vol. 41. - Article ID 412. - DOI: 10.1007/s40430-019-1917-2.

11. Burmasheva N. V., Prosviryakov E. Yu. An exact solution for describing the unidirectional Marangoni flow of a viscous incompressible fluid with the Navier boundary condition. Temperature field investigation // Diagnostics, Resource and Mechanics of materials and structures. - 2020. Iss. 1. - P. 6-23. - DOI: 10.17804/2410-9908.2020.1.006-023 . - URL: https://dreamjournal.org/DREAM_Issue_2_2020_Burmasheva_N.V._et_al._061_075.pdf

12. Burmasheva N. V., Prosviryakov E. Yu. Exact solution for describing a unidirectional Marangoni flow of a viscous incompressible fluid with the Navier boundary condition. Pressure field investigation // Diagnostics, Resource and Mechanics of materials and structures. - 2020. - Iss. 2. P. 61-75. - DOI: 10.17804/2410-9908.2020.2.061-075. - URL: https://dreamjournal.org/DREAM_Issue_2_2020_Burmasheva_N.V._et_al._061_075.pdf

13. Kinderlehrer D., Stampacchia G. An Introduction to Variational Inequalities and Their Applications. - New York : Academic Press, 1980. 